\title{
LIGHTWEIGHT HEADGEAR BRAIN WAVES FOR FATIGUE DETECTION IN SMARTPHONE
}

\author{
Yung Gi Wu, Jwu Jenq Chen and Rui Hsin Wang \\ Department of Computer Science \& Information Engineering, \\ Chang Jung Christian University, Taiwan.
}

\begin{abstract}
Fatigue is an annoying phenomenon that can affect people's concentration on learning or working. It will reduce the learning efficiency of people and even cause danger in working. For example, fatigue driving results in car accident have been reporting from the daily news. In order to avoid the accidents mentioned above, this research design the mobile system to detect fatigue to alert people by way of the lightweight brainwave detector, and the smartphone that everyone owns nowadays. In this system, light weight head mounted brainwave device is adopted and the signal are transmitted to the smartphone for further processing. Our algorithm calculates the fatigue index by focus, eyes blink frequency, $\delta$-wave, $\alpha$-wave, $\beta$ wave, and $\theta$-wave captured from the brainwave to determine human's spiritual condition. When the experimental results were carried out to the car drivers, the system can remind drivers when they were tired and drowsy while driving. This system can notify people to aware his own spiritual state so as to raise working efficacy and avoid the occurrences of accident caused by fatigue. In addition, the whole system without complex instrument and expensive cost.
\end{abstract}

\section{KEYWORDS}

Brainwave Device, Fatigue Detecting, Smartphone, headgear

\section{INTRODUCTION}

Fatigue is a feeling of extreme mental and physical tiredness. The causes to fatigue could be disease, long-term activities, drug treatment, pain, muscle weakness, over-doing, low-mood, poor diet, hunger...etc. Most of us feel tired in monotonous activity or a long time working and people can recover and improve this kind of fatigue after rest. Therefore, many companies or factories make regulation to let the employees to rest after a period time of working and that can raise company's productivity and decrease the risk of operation caused by fatigue. However; some jobs don't allow employees to take a break in regular period, such as long trip drivers.

3,000 4,000 people die on the roads every day in the world and tens of millions of people are injured or disabled every year according to report from WHO[1]. Road traffic crashes will become the seventh cause to make people to die in the future. Traffic Safety at the American Automobile Association (AAA) announced that driver's fatigue is the second biggest factor to cause road accident [2]. It leads to 100,000 car accidents every year in the USA. Most risky driving time are between 11 p.m. to 8 a.m. For most people, our circadian rhythms tell our bodies 
that it's time for sleep during that time just mentioned above. In addition, long distance driving and take drug are all the causes to drowsy. Fatigue is fatal in some scenarios.

In general, fatigued people experience frequent yawning, heavy eyelids, misjudging operation, In general, fatigued people experience frequent yawning, heavy eyelids, misjudging operation, fluctuating seeing, daydreaming... etc. From the literature, we know the methods to detect fatigue, such as eye movement patterns, face recognition analysis, and monitoring the breathing and heart rate. The technology for detecting fatigue driving have been researching to raise the safety of people and car. Therefore, some cars equipped with the fatigue detection system. They analyse various clues, including steering behavior, pedal usage, and vehicle acceleration, to automatic assess the driver's driving mental or physical states. If the system detects that the driver may be in a state of insufficient attention, a warning light from the coffee cup will be displayed on the driver's screen to remind him to resting for a while. Some cars accumulate the driving times to notify the driver to rest to recover his attention. Most of the detection system judge the state of the driver by his external behaviours. In this paper, we detect the brainwaves of person and analyse the signal it outputs to acquire current mental or body state. The fatigue index is displayed on the mobile phone. If it exceeds the alarm threshold for more than 5 seconds, it will alert by sounding alarm. At the same time, the system establishes a database to store the data after brainwave analysis to provide users with a history for querying, to know when they are prone to fatigue to avoid accident during periods of easy to be fatigue so as to reduce the risk of accidents. his fatigue detection system can be used for the driver, students or operator. In the following, we will depict some products that are adopted in real world now.

First of all, this research compares the existing fatigue-related methods. Japan, Australia, and Spain all have been researching on detecting fatigue for various kinds of operators. Then, we survey several available commercially brainwave meters and compare in terms of cost, accuracy, ease of carrying (mobility), ease of operation for comprehensive evaluation to select the proper brainwave meter instrument that meets the our requirements.

Japanese eyewear manufacturer JIN published a glasses product JINS MEME on 2014 [3]. It measures the eight directions of the eyeball by using the electrooculography sensing technology. The potential difference caused by changes in the eyeball and the movement of the eye in all directions can be detected and calculated to determine the direction of movement of the line of sight and other information. The weight of this glasses is about 40 grams. A little heavier than the glasses that most people wear. Not all users have the habit of wearing this kind of glasses.

A seeing machines company in Canberra, Australia, designed and developed the Fatigue Monitoring System for mine truck drivers in 2013 [4]. The size used for the mine truck driver is so large that it can easily cause staff to be masked by the vehicle next to him. This system consists of a set of infrared cameras that can be detected even when driving with sunglasses that cover the eyes. It also includes an image processor that records the duration, frequency, and speed of eyes blink to evaluate if distraction or involuntary fragment sleep happened. Once the system determines that the mental condition is poor, the chair not only makes strong vibrations but also sounds harsh alarms. However, if the driver's eyes cannot be detected by the camera because of occasional bowing his head, the alarm will sound as well.

The Valencia Institute of Biomechanics in Valencia developed the "HARKEN" smart seat belt, which detects the breathing and heartbeat frequency of driving and warns in times of crisis; this 
equipment consists of three main parts, first the seat belt The heartbeat sensor is followed by a breathing detector near the back of the chair, and finally the signal processing unit "SUP" under the seat, which is responsible for analysing the data of all electronic detectors [5]. Endurance athletes, whose heartbeat is lower than the normal people may make the smart seat belt misjudge.

At the 2014 Consumer Electronics Show (CES) in Las Vegas, Xuan Rui Technology from Taiwan demonstrated a device called U-Wake, a standard human-machine interface wearing device that can detect dangerous driving through the change of brainwaves. The brainwave fatigue detector uses brain-computer interface technology. The degree of user fatigue can be instantly displayed on the mobile phone's APP, and reminds the user to stay awake [6], but the system is very expensive.

The above four products are available in the market to remind the mental or physical fatigue state for the operator during his operation. We depict various kinds of brainwave-meter instruments in the following. The first one is the traditional headgear device. When performing brainwave measurement in traditional way, people should wear a special headgear as shown in Figure 1 to collect the signal. There are multiple electrode pads in the headgear to contact the scalp, and the scalp should be wet-adhesive. The test site must be manipulated in a specific and non-interfering environment, such as in hospital. It is difficult for car drivers or mechanical operator's usage.

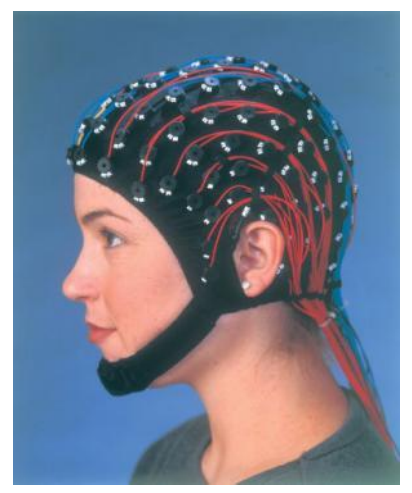

Figure 1. Headgear style brainwave meter [7]

The second whole brain covered high density brainwave instrument by dEEG. dEEG is a medical device company that uses the most advanced EEG technology to create a brain-covered highdensity brainwave instrument as shown in Figure 2[8]. It has accurate and convenient recording system. In addition, it has 32, 64, 128 or 256 channels to select. The operator of this instrument is required to have the original and complete training to operate it correctly. It is not suitable for general usage either.

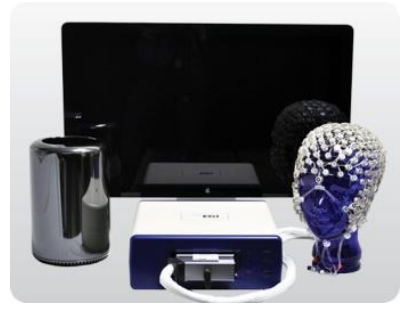

Figure 2. Whole brain covered high density brainwave instrument by dEEG[8] 
The third one is mindwave mobile starter brain cube as shown in Figure 3 [9]. It is a lightweight research-grade EEG headset. Wear it like listening to music with headphones so that it is suitable for various kind of operators to use. The embedded bio-sensor chip can read EEG signalsby noninvasive dry electrodes, filter out nearby noise and electrical interference, and convert the measured signals into digital signal [10].Measured data obtained through this device contain raw brainwaves, concentration, blink detection, relaxation and even more including detecting whether the device is worn in poor contact. These data can assist to analyse brainwaves in relatively simple way and develop a variety of applicable functionalities. This device owns the advantage of easy to carry and highsensing precision. Moreover; the cost of this equipment is only a small portion of research-grade equipment but it reaches $96 \%$ accuracy compared to research-grade instrument.

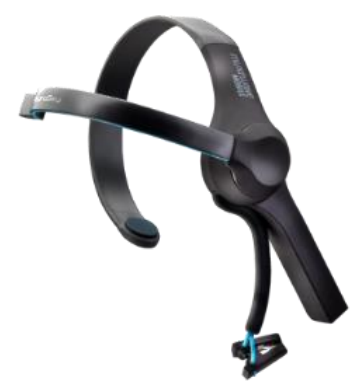

Figure 3. Mindwave mobile starter brain cube

Table 1 shows the comparisons among the three brainwave instruments depicted above.

Table 1 Comparisons of brainwave meters

\begin{tabular}{|l|l|l|l|}
\hline Type & $\begin{array}{l}\text { Traditional } \\
\text { headgear style }\end{array}$ & $\begin{array}{l}\text { Whole brain } \\
\text { covered high } \\
\text { density style }\end{array}$ & $\begin{array}{l}\text { Mobile starter } \\
\text { brain cube }\end{array}$ \\
\hline cost & high & high & low \\
\hline precision & high & high & high \\
\hline mobility & no & no & yes \\
\hline $\begin{array}{l}\text { operation\& } \\
\text { manipulation }\end{array}$ & complicated & $\begin{array}{l}\text { Professional } \\
\text { training }\end{array}$ & easy \\
\hline $\begin{array}{l}\text { conditions for } \\
\text { measurements }\end{array}$ & $\begin{array}{l}\text { undisturbed } \\
\text { environment }\end{array}$ & uncomfortable & unlimited \\
\hline
\end{tabular}

After considering all perspectives, we adopt mobile starter brain cube to be our brainwave meter instrument.

In [10], they conducted a study to measure the changes in mental workload of EEG in a noninvasive manner situation. Based on the simulation of the driving platform, the brainwave is used to analyse and estimate the driver's driving situation. Time-frequency analysis and other technologies are used to construct a system to estimate the driver's psychological state by brainwaves. In [11], it's a study on driver's brainwave cognitive response estimation through non-invasively recorded brainwave analysis to identify the human brain's response to event stimuli and to estimate the driver's spiritual alert level to dynamically measure the driver's mental state change, and simultaneously measure the corresponding cognitive, vehicle control and 
driving behaviour changes to maintain the driver's in good spirt mode to prevent driving accidents in driving. In [12], it used EEG components to evaluate the algorithm for detecting fatigue. It confirmed that the ratio of slow wave to fast wave in EEG activity increased with time, and the results of this study have the significance of detecting fatigue. In [13], it claimed that as a person fatigues, the brain loses capacity and slowdown his activity behaviour, and that attempts to maintain vigilance levels leading to increased $\beta$ wave activity. According to the literature survey, most of the researches are in the academic field of biological category. They research and find out relationship between the brainwave and fatigue condition. However, no one uses the brainwave data to make a convenient device system for users to measure fatigue while operating or working. In this research, we detect and driver's fatigue and record the brainwave data in the database. We can not only detect the fatigue condition but also recording the data for long term observation in easy and convenient way.

In this paper, we adopt SQLite to record the data. SQLite is an open source database library, and complies with the standards of ACID-related database. It uses a standard SQL syntax to provide a stand-alone database system that does not require a connecting to environment. It is widely used in various operating systems, embedded systems and browsers. Android supports the SQLite database, so that each application can create its own database, store the data in the SQLite database file, the developers can use the API library provided by Android to access the data in the database for specified applications, including query, add, delete and update standard SQL access syntax, it can also set the version of the database, you can also copy and migrate the database in the new version, so we chose it to build database.

\section{The Proposed METhod AND SySTEM Establishment}

\subsection{Brainwave detection}

The whole system comprises two major stages. The first stage is to collect the signals from mobile starter brain cube meter. The signals are transmitted to the smartphone to process by Bluetooth. Brainwave analysis and recording in the smartphone are the major topics in the second stage. The brainwave acquired by brain cube meter is divided into different bands by Fourier transform. This device has embedded an eSense algorithm that can generate the following data: original brainwave signal, concentration, relaxation indices, brainwave band data $(\delta, \theta, \alpha, \beta)$ and blink detection. Physical and psychological conditions represented by various frequency bands of brainwaves can be found easily from web. The general properties and frequency ranges of the $\delta, \theta, \alpha, \beta$ waves are given in Table 2[14].

Table 2 Brainwaves properties and frequency ranges

\begin{tabular}{|l|l|l|}
\hline Brainwaves & Frequency & Property \\
\hline Delta $(\delta)$ & $0.1 \sim 3 \mathrm{~Hz}$ & $\begin{array}{l}\text { Unconscious condition. } \\
\text { Occurs during the third phase of non-rapid eye } \\
\text { movement sleep. }\end{array}$ \\
\hline Theta $(\theta)$ & $4 \sim 7 \mathrm{~Hz}$ & $\begin{array}{l}\text { Denote subconscious condition } \\
\text { There are memories, perceptions and } \\
\text { emotions. } \\
\text { Affect attitudes, expectations, beliefs, } \\
\text { behaviors. }\end{array}$ \\
\hline
\end{tabular}




\begin{tabular}{|c|c|c|c|}
\hline & & & $\begin{array}{l}\text { The source of creativity and inspiration. Deep } \\
\text { sleep, deep meditation. } \\
\text { Psychic awareness, strong personal } \\
\text { knowledge, strong personality }\end{array}$ \\
\hline \multirow[t]{3}{*}{$\operatorname{Alpha}(\alpha)$} & Low range & $8 \sim 9 \mathrm{~Hz}$ & $\begin{array}{l}\text { A state of mind before going to sleep. } \\
\text { Consciousness is gradually drowsy. }\end{array}$ \\
\hline & $\begin{array}{l}\text { Middle } \\
\text { range }\end{array}$ & $9 \sim 12 \mathrm{~Hz}$ & $\begin{array}{l}\text { Inspiration, intuition or idea raising. } \\
\text { Relaxed but focused }\end{array}$ \\
\hline & High range & $12 \sim 14 \mathrm{~Hz}$ & Highly alert. \\
\hline \multirow[t]{3}{*}{$\operatorname{Beta}(\beta)$} & Low range & $12.5 \sim 16 \mathrm{~Hz}$ & Relax but concentrated. \\
\hline & $\begin{array}{l}\text { Middle } \\
\text { range }\end{array}$ & $16.5 \sim 20 \mathrm{~Hz}$ & Thinking, dealing with external messages. \\
\hline & High range & $20.5 \sim 28 \mathrm{~Hz}$ & Excitement, anxiety \\
\hline
\end{tabular}

The brainwave used to represent the body health can be brief depicted as follows. $\delta$ wave: rest wave. Deep sleep state will appear generally in high value. $\theta$ wave: repair wave. Extremely relaxed, also known as the Buddha brainwave, strong repair. awave: healthy wave. Relax brainwaves, high immune status, secrete morphine in the brain, and have self-healing ability. $\beta$ wave: sick wave. Nervous brainwaves, low immunity, easy to get sick.

The TGAT chip in the mindwave mobile starter brain cube, which is a highly integrated system single-chip brain inductor that can perform analogy-to-digital conversion, which can detect abnormalities in poor contact, and filter out abnormal eye movements and $50 \mathrm{hz}$ and $60 \mathrm{hz} \mathrm{AC}$ signal interference in circuit; eSense is an algorithm that is capable of measuring the mental state and collects the user's concentration index and blink strength. In this research, the four frequency bands signal $(\delta, \theta, \alpha, \beta)$, concentration index and the blink strength are transmitted by Bluetooth to smartphone for further fatigue detection and stored for long term observation.

\subsection{SQLite database establishment}

We develop an APP that can be installed in smartphone to increase the mobility of this system. APP is a convenient way to any end users to use because it has the advantage of mobility. For the diver, all he has to do is to install the APP in his smartphone and open this APP while driving. Of course, he must wear the brain cube in his head. The following section will give you a brief depict about our SQLite database design in smartphone side. We adopt the Android Studio to implement the system including user interface, Bluetooth connection and SQLite database.

Note that the user must register an account to keep his own data. The historic data can be observer by user to know his own mental condition in every driving. If his friend wants to use this smartphone to raise the driving safety, his friend can register another account.

In this the research, the whole project has six activities named Main Activity, register, Bluetooth, show_result, record and Record DBHelper. The functions are the main page, registration screen, Bluetooth connection screen, display measurement data screen, history record andlayout respectively. Below figure shows the project structure (Figure 4). 


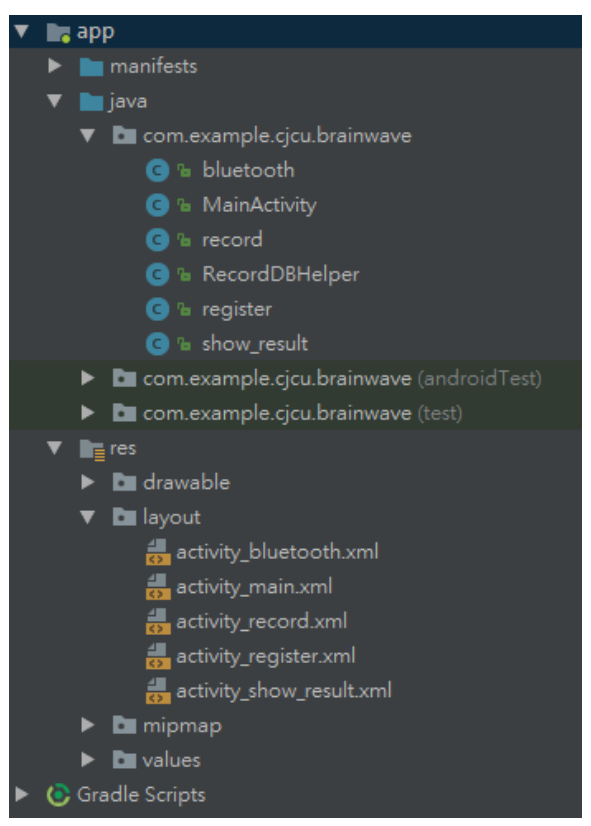

Figure 4.Project configuration of this APP

The database created by SQLite is used to store the user's basic data and usage records. The project first creates a database named "brainwaves". The database contains two data tables named "User" and "Data" (Figure 5). "User" table includes_id, name, gender, age, phone, account and password. The "Data" data table is used to store the relevant data of the driver's use process. The nine fields includes _id, date, time, alpha, beta, delta, theta, focus and wink. The primary key is _id. Note that the value ranges of alpha, beta, delta, theta, focus and wink are from 1 100.

\begin{tabular}{|c|c|c|c|c|c|c|c|c|}
\hline $7 \quad$ id & name & gender & age & phone & account & password & & \\
\hline \multicolumn{9}{|c|}{ table of Data } \\
\hline id & date & time & alpha & beta & delta & theta & focus & wink \\
\hline
\end{tabular}

Figure 5. Brainwaves database fields

Use SQLite to add DATABASE named "brainwave". Create a "user" data table under "brainwave", and in the on Create method, after getting the SQLite Database object, call the execSQL method to create a "user" data table. Using the above method in "brainwave" to create a "data" datatable under the "brainwave" database by calling the execSQL.

When the user registers, system will confirm whether the data entered by the user are all filled in. The name, gender, age, mobile phone number, account number and password must not be null before the file can be written. Using getWritableDatabase() to construct the database, ContentValues values $=$ new ContentValues() to store the data to be added, and db.insert() to write the data to the database. Figure 6 shows how they work. 


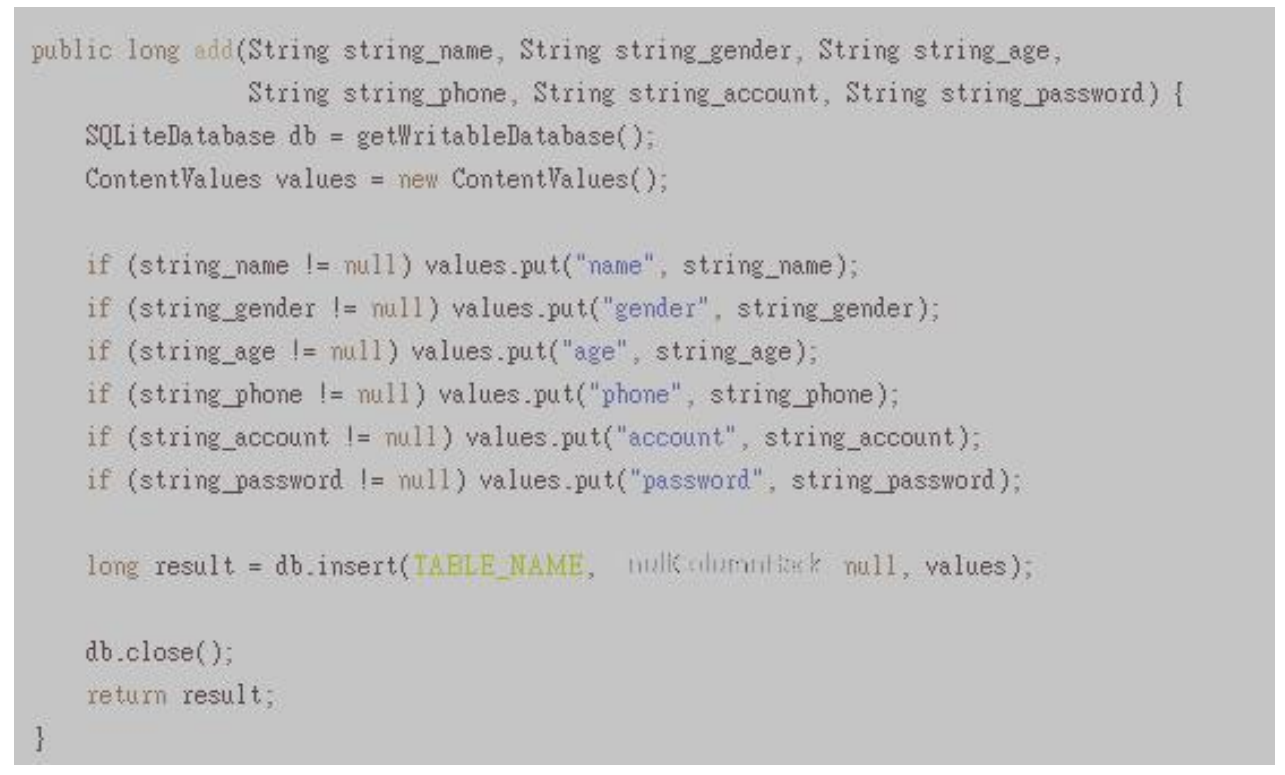

Figure 6. Program codes for user registration

\subsection{Fatigue detection criteria}

Common features of fatigue include: body and limbs feeling heavy and difficult to move, flu-like feelings of exhaustion, eyelids are heavy, feeling energy has drained away slowly. When fatigue is severe, it can lead to feelings of energy complete exhaustion, or 'wipe-out'. Then, you have to sit or lie down to try to recover. Otherwise, it can lead to dangerous no matter in driving or operating machine. For the students, it can obstruct the learning in classroom or self-learning.

In this paper, aiming at our goal to detect fatigue before body physical lie down. The mindwave mobile starter brain cube reads the brainwave in the sampling rate of $512 \mathrm{~Hz}$. But, we just take one data per second in case occupying too much data storage in smartphone. After the data is written into the database, the obtained $\delta, \theta, \alpha$ and $\beta$. Note that $\alpha$ and $\beta$ values that we acquire from brainwave are $\alpha \_$mid and $\beta$ _low which presenting the awaken state. However; the middle frequency of $\alpha$ wave $\left(\alpha \_\right.$mid) and the low frequency of $\beta$ wave ( $\beta \_$low) are the representative values of focus and concentration. While the $\delta$ and $\theta$ waves denote the unconscious state. Under the brainwave pattern, the purpose of this project is to detect the degree of fatigue by measuring brainwaves under awake conditions. Therefore, the $\alpha \_$mid and $\beta$ low represent the sobriety and concentration are placed in the denominator, $\delta$ and $\theta$ represent the unconscious state are placed in the numerator. If a person is feeling fatigued or drowsy, his eye blinking pattern changes that is varied from the average blinking period. When we wear this device, we calculate the averaged wink (winkavg) value in the first minute. Therefore, the signal of focus and blink values (wink) are integrated together also to obtain the fatigue index as in (1).

$$
\text { fatigue_index }=\frac{\delta+\theta}{\alpha_{\min }+\beta_{\text {low }}} * w 1+\frac{1}{\text { focus }} * w 2+\mid \text { wink }-w i n k_{\text {avg }} \mid * w 3(1)
$$

Higher fatigue_index denotes the mental or physical state is drowsy. If the fatigue indices exceed the threshold value and lasting for the time that we set, the APP in the smartphone will sound alarm to remind us to be careful or rest to avoid any dangerous event happened. The parameters $(w 1, w 2, w 3)$ in $(1)$ are set to $(10,5,5)$ after repeated experiments. 


\section{EXPERIMENTAL RESUltS}

Fatigue is one of the significant factors to cause harm, accidents, and hazard in industries. Especially to the jobs such as mining, transportation, construction, manufacturing and healthcare. To the students in classroom or the employees in company, fatigue obstructs the learning and working efficiency as well. In order to avoid the occurrences of dangerous during the experiments, we exclude experiments that lead to a fatal crisis to carry out. The students, employees in office and the driver are invited to participant the experiments.

Most of the people feel drowsy after lunch; therefore, most of the companies provide one or two hours rest time to the employees to lunch and take a nap. Twelve students and eight employees in office whose ages are from 18 to 37 took part in the experiment. The fatigue-index threshold to alert alarm we set in this research is 40 . They were requested to take the lightweight brainwave device after lunch. Two of them felt uncomfortable because the device must clamp to the left ear lobe while wearing it so as to fail to the test. The other eighteen participants were reminded by smartphone when they felt drowsy to sleep.

Next experiment was kind of risky. Because fatigue driving harms to human life seriously, we made the experiment to drivers. In this test, ten people whose ages are from 20 to 50 were invited to participant this research. The driving time was from pm 10:00 to 11:00 because circadian rhythms tell our bodies that it's time for sleep for normal people. Nine people completed the test. Seven of them agreed that it had a remind effect. Two of the testers who are young drivers thought that there was no feeling about this system. They are accompanied by friends and chatted with them while driving. One tester claimed that she was not used to reacting with this device because of the clamping to the left ear lobe, she felt uncomfortable and could not finish the test.

Figure7 illustrates the fatigue-index curve of a tester who is 50 years old man. He was asked not to turn on the radio in car and driving alone. There are 3600 seconds in one hour recording. Those values that are suddenly abnormally high in the earlier time could be the device error. It could be the error caused by poor contact of the measuring point or the light from the opposite road. When the fatigue-index exceeded the threshold for more than 5 seconds, it alerted the driver by sounding alarm. It can be observed that he could only concentrate on driving at the early stage.

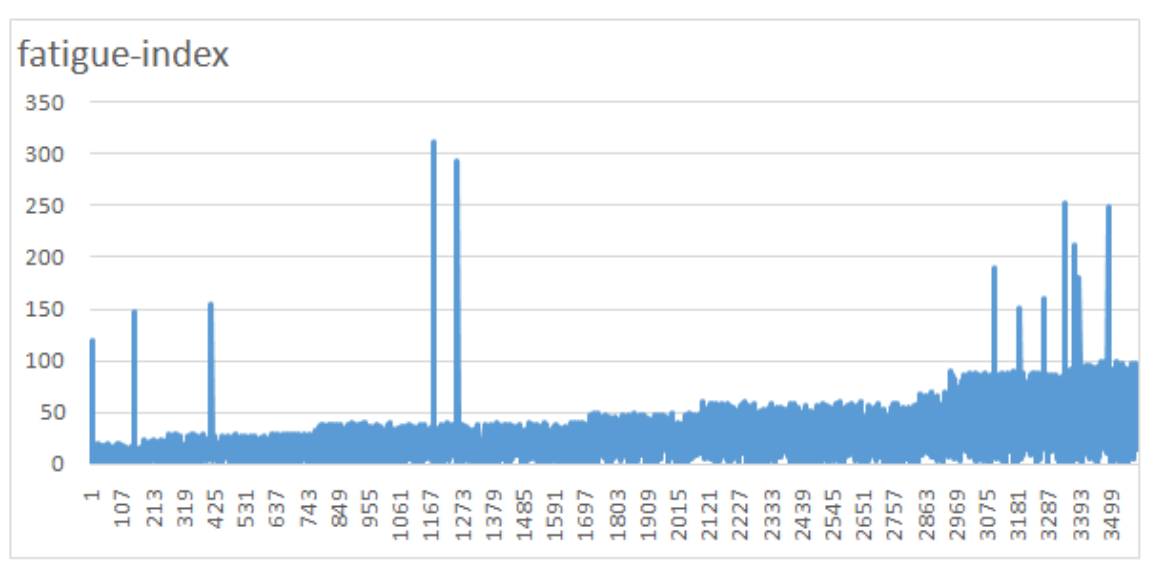

Figure 7. Fatigue-index data for 3600 seconds 
International Journal of Computer Science \& Information Technology (IJCSIT) Vol 11, No 5, October 2019

To sum up, twenty five testers were reminded when they felt fatigue successfully. The experiments demonstrated that the system can remind people when they felt drowsy. In addition, even though the device is lightweight but it need to clamp to the left ear lobe, some of testing people experienced it uncomfortable.

\section{CONClusion}

Fatigue could be a fatal crisis in real world. It is a mental or physical tired state for human so as to cause dangerous to operating machine or obstruct the learning or working efficiency. Some researchers have been carrying out to the apparent behaviours analysis to detect the fatigue; however, it could be too late once the apparent behaviours happened. This research adopt brainwave to detect fatigue and hope to remind people before crisis occurred. Most of brainwave measure instruments are expensive and professional operators are required to operate them. In addition, they are not easy to wear to head so that they are suitable for general usage. In this paper, we use a lightweight brainwave device which is easy to use and wear to head to detect fatigue. Experiments demonstrated that it's capable to detect the fatigue for school students and employees in company. Furthermore, the crisis of injury caused by fatigue driving is getting more and more serious. When big accident occurred, the government institute always review driver's driving regulation, such as forcing the tour bus driver to rest after driving one to two hours. However; the physical fitness and mental state of each driver are different. We don't know whether the driver take drug or drink wine before driving. Even enough resting time, fatigue driving still may occur. Therefore, the most important thing to avoid fatigue driving is immediate detection and reminding. The brainwave detection driving fatigue system built by the research can remind the driver by sounding alarm when fatigue occurs. Drivers only need to buy a headgear brainwave and smartphone then equipping this APP. The system has two features of low cost and easy to operation which can raise the user's willingness to purchase and use. We want to encourage drivers to adopt this technology and use it to decrease accidents caused by fatigue. As to the further development, the brainwave device and the hat can be integrated on the hardware to facilitate the driver's willingness to use. The long term historic record can also be applied to other mental-related issues.

\section{ACKNOWLEDGEMENTS}

The authors would like to appreciate the partial support from the Minister of Science \& Technology, Taiwan under the project no. 106-2813-C-309-002-E. The preliminary paper was presented in the ICAIT 2019 [15].

\section{REFERENCES}

[1] World Health Organization. (2018).Road traffic injuries. Available at: https://www.who.int/violence_injury_prevention/road_traffic/en/ [Accessed 10 Nov. 2018].

[2] Collision, W. (2018).The Leading Causes of Car Accidents - Causes and Statistics. Waterdown Collision. Available at: https://www.waterdowncollision.com/blog/safe-driving/leading-causes-ofcar-accidents/ [Accessed 10 Nov. 2018].

[3] JINS MEME. (2018).JINS MEME: The world's first wearable eyewear that lets you see yourself. Available at: https://jins-meme.com/en/ [Accessed 10 Nov. 2018]. 
International Journal of Computer Science \& Information Technology (IJCSIT) Vol 11, No 5, October 2019

[4] Seeing Machines. (2018).Driver monitoring technology for automotive, transport \& logistics industries. Available at: https://www.seeingmachines.com/ [Accessed 10 Nov. 2018].

[5] Harken.ibv.org. (2018).Wouldn't you want an alarm to go off if you fall asleep driving?. Available at: http://harken.ibv.org/ [Accessed 10 Nov. 2018].

[6] Digital Trends. (2018).U-Wake | Wearable brainwave sensor alerts fatigued drivers | Digital Trends. Available at: https://www.digitaltrends.com/cars/u-wake-wearable-brainwave-sensor/ [Accessed 11 Nov. 2018].

[7] Evolving Science. (2017).Monitoring the Brain Outside the Lab. Evolving Science. Available at: https://www.evolving-science.com/bioengineering-brain-computer-interfaces/monitoring-brainoutside-lab-00129 [Accessed 11 Nov. 2018].

[8] Nutronic Techtronic Co. (2018).dEEG whole brain covered high density brain wave. Available at: http://nutronicltd.com/?deeg\%E5\%85\%A8\%E8\%85\% A6\%E8\%A6\%86\%E8\%93\%8B\%E5\%BC\%8 F\%E9\%AB\%98\%E5\%AF\%86\%E5\%BA\%A6\%E8\%85\%A6\%E6\%B3\%A2\%E5\%84\%80, 177 [Accessed 11 Nov. 2018].

[9] NeuroKky. (2018).EEG Headsets | NeuroSky Store. Available at: https://store.neurosky.com/ [Accessed 12 Nov. 2018].

[10] Teng-Yi Huang,"Development of dynamic VR driving platform and its application on driver's cognitive state estimation,"Master dissertation, Dept. Elect. \& Ctrl. Eng., National Chiao Tung Univ., Hsinchu City, Taiwan, 2003.

[11] Ruei-Cheng Wu, "EEG-Based Assessment of Driver Cognitive Responses and Its Application to Driving Safety," Ph.D. dissertation, Dept. Elect. \& Ctrl. Eng., National Chiao Tung Univ., Hsinchu City, Taiwan, 2005.

[12] Jap, B. T., Lal, S., Fischer, P., \&Bekiaris, E., (2009)“Using EEG spectral components to assess algorithms for detecting fatigue", Expert Systems with Applications,Vol. 36, No. 2, pp2352-2359.

[13] Craig, A., Tran, Y., Wijesuriya, N., \& Nguyen, H., (2012)“Regional brain wave activity changes associated with fatigue", Psychophysiology, Vol 49, No 4,pp574-582.

[14] Wikipedia. (2018).Brainwave - Wikipedia, the free encyclopaedia. Available at: https://zh.m.wikipedia.org/zh-tw/\%E8\%85\%A6\%E6\%B3\%A2 [Accessed 12 Nov. 2018].

[15] Yung Gi Wu, JwuChenq Chen, RuiHsin Wang, 'The System Design for Fatigue Driving Detection by Brainwaves Analysis in Smartphone', 8th International Conference on Advanced Computer Science and Information Technology (ICAIT 2019), Mar. 2019 , pp. 1-11 ,Zurich.

\section{AUTHORS}

Yung Gi Wu. He received $\mathrm{Ph}$. D. degree from the Institute of Electrical Engineering. National Cheng Kung University. Tainan, Taiwan, 2000. He is now a professor with the Department of Computer Science \& Information Engineering. Chang Jung Christian University in Taiwan. His research fields includes data compression, optimization, information retrieval, digital signal processing, AI\& IoT applications.

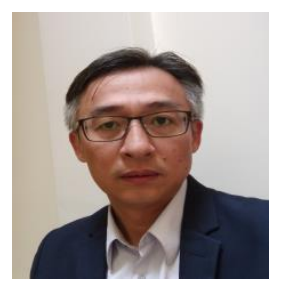


International Journal of Computer Science \& Information Technology (IJCSIT) Vol 11, No 5, October 2019

Jwu-Jenq Chen was born in Tainan County in Taiwan. He obtained a MS degree from the Graduate Institute of computer science, Ohio University in U.S.A., 1996 and then received a Ph.D. from the Graduate School of Business and Operations Management, Chang Jung Christian University in Taiwan.He had worked as technician and supervisor on computer system for 13 years. At this time, he is anAssociate Professor at the department of Computer Science and Information Engineering at the Chang Jung Christian University in Taiwan. Hisresearch fields includes management information system and website.

Rui Hsin Wang. She received the bachelor degree from the Department of Computer Science and Information Engineering at the Chang Jung Christian University in Tainan, Taiwan, 2017. She is now a student in the Institute of Information Engineering. National Chung Hsing University. Taichung. Taiwan.Her research fields includes digital signal processing and mobile computing.
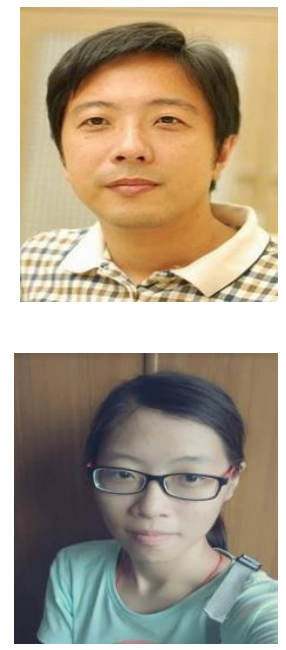